\title{
Extreme Learning Machine Approach for Real Time Voltage Stability Monitoring in a Smart Grid System using Synchronized Phasor Measurements
}

\author{
P. Duraipandy ${ }^{\dagger}$ and D. Devaraj*
}

\begin{abstract}
Online voltage stability monitoring using real-time measurements is one of the most important tasks in a smart grid system to maintain the grid stability. Loading margin is a good indicator for assessing the voltage stability level. This paper presents an Extreme Learning Machine (ELM) approach for estimation of voltage stability level under credible contingencies using real-time measurements from Phasor Measurement Units (PMUs). PMUs enable a much higher data sampling rate and provide synchronized measurements of real-time phasors of voltages and currents. Depth First (DF) algorithm is used for optimally placing the PMUs. To make the ELM approach applicable for a large scale power system problem, Mutual information (MI)-based feature selection is proposed to achieve the dimensionality reduction. MI-based feature selection reduces the number of network input features which reduces the network training time and improves the generalization capability. Voltage magnitudes and phase angles received from PMUs are fed as inputs to the ELM model. IEEE 30-bus test system is considered for demonstrating the effectiveness of the proposed methodology for estimating the voltage stability level under various loading conditions considering single line contingencies. Simulation results validate the suitability of the technique for fast and accurate online voltage stability assessment using PMU data.
\end{abstract}

Keywords: Extreme learning machine, Loading margin, Voltage stability assessment, Phasor measurement unit

\section{Introduction}

With the continuous increase in power demand over the years, the present day power systems remain highly stressed and tend to operate very close to the point of voltage collapse. A gradual increase in system loading, followed by a severe contingency may thus lead to voltage instability, which may ultimately lead to voltage collapse causing blackouts. Hence, developing a fast and accurate computational tool for real time voltage stability monitoring is very much essential for the upgradation of present power grid to a future smart power grid.

Voltage stability can be assessed using static and dynamic methods. Static voltage stability analysis is commonly used for clear examinations of a wide range of system conditions and to identify the key contributing factors. Static method of voltage stability analysis is done using power flow results by solving algebraic equations. Time domain simulation method is used for dynamic voltage stability analysis by solving differential equations.

Many analytical methods have been proposed in the

$\dagger$ Corresponding Author: Dept. of Electrical and Electronics Engineering, Velammal College of Engineering \& Technology, India. (vai 2k4@yahoo.co.in)

* Dept. of Electrical and Electronics Engineering, Kalasalingam University, India. (deva230@yahoo.com)

Received: December 30, 2014; Accepted: July 9, 2016 literature [1-12] for the static and dynamic method of voltage stability assessment so far are time consuming which limits their application for on-line. To overcome the limitation of conventional voltage stability assessment method, Artificial Neural Networks (ANN) trained by traditional learning algorithms have been proposed [13][19] for voltage stability assessment. Because of the slow gradient-based learning algorithms with all the parameters tuned iteratively, the training time of feed forward neural network is in general higher. Also, the conventional learning algorithms suffer from slow convergence, local minima and over-fitting problems. Further, it involves too many parameters which are needed to be tuned randomly.

This paper presents an Extreme Learning Machine (ELM) approach for loading margin estimation under multiple contingencies using real-time measurements from PMUs. PMU provides precise estimates of the power system state at frequent intervals through GPS synchronization. Synchrophasor data can help the operators in the control centre to take corrective measures by providing an early and improved detection of evolving grid problems. Also, PMU provides time synchronized phasor data at high sampling rate for an efficient monitoring of voltage stability in real time to improve the security of the smart power grid. The placement of PMU at suitable location is an important task in the Smart grid. PMUs are located in 
such a way that they provide optimal measurements to achieve the full network observability with minimum number of measuring devices. Depth First (DF) search method is used for placing the PMUs. To make the ELM approach applicable for a large scale power system problem, MI-based feature selection is proposed to achieve the input dimensionality reduction. MI-based feature selection reduces the number of network input features which reduces the network training time and improves the generalization capability. Voltage magnitudes and voltage angles obtained from PMUs by the simulation results are fed as inputs to the ELM model. Loading margin is a good indicator for assessing the voltage stability level which is the output of the network. IEEE 30-bus system is considered for demonstrating the effectiveness of the proposed methodology under various loading conditions considering single line contingencies.

\section{Computation of Loading Margin}

Loading margin is a good indicator for assessing the voltage stability level of the power system. The amount of load increase from the base load point to the maximum loading condition without voltage collapse, according to the system loading parameter $\lambda$ is known as loading margin or voltage stability margin. The loading parameter is 0 for base loading condition and $\lambda_{\max }$ for the maximum loading condition. The maximum loading parameter $\lambda_{\max }$ gives the loading margin as shown in Fig. 1. Loading margin is computed from the nose curve using Continuation Power Flow (CPF) method. The conventional power flows do not converge at the maximum loading point where the Jacobian matrix becomes singular. Continuation power flow overcomes this problem by reformulating the power equations by introducing an additional parameter. It includes two steps, namely, prediction and correction steps. In the first step, for a certain increase in load a new solution is predicted from a known base solution. In the second step, the exact solution is calculated using NewtonRaphson power flow. Both the steps are repeated until the tangent vector becomes zero and maximum loading point is reached with the help of which loading margin is calculated. Thus the CPF method of obtaining the loading

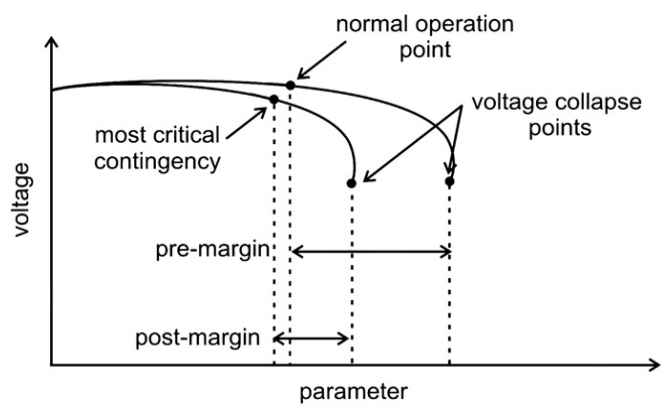

Fig. 1. Loading Margin margin is a time consuming process which limits its application for online. Fig. 1 shows the pre-loading margin obtained during normal operating condition and post-loading margin after the occurrence of a critical contingency. In this work, ELM network is proposed to quickly estimate the loading margin using real time measurements from the PMUs.

\section{Placement of PMU}

The input data for the proposed online voltage stability monitoring system are the voltage magnitudes and the phase angles which are obtained from PMUs by the simulation results. The placement of PMU at suitable location is an important task in the Smart grid. PMUs are located in such a way that they provide optimal measurements to achieve the full network observability with minimum number of measuring devices. In this section, method of PMU placement is discussed with the aim of linear static state estimation of power system networks. There are various methods available for PMU placement in literature [20]. Among these methods, Depth First (DF) search method is adopted for placing the PMU.

\subsection{Rules for PMU placement}

The PMU placement rules proposed in [20] are adopted in this work and are reproduced below:

1. One voltage measurement is assigned to a PMU placed bus including one current measurement to each branch connected to it.

2. One voltage pseudo- measurement is assigned to each bus connected to another PMU equipped bus.

3. One current pseudo- measurement is assigned to each branch connecting two PMU placed buses.

One current pseudo-measurement is assigned to each branch where current can be indirectly calculated by the Kirchhoff current law (KCL).

\subsection{Depth first method of PMU placement}

Depth First method (DF) is one of the tree search methods of PMU placement. This method uses only first three rules of PMU placement. The essence of this method expands from the nodes placed the PMU to the pseudomeasurement voltage nodes through the measurement or pseudo-measurement current branches, and then to all the nodes. The expanded nodes create a metrical tree, if the tree contains all the node of the system, then the system is topology observability, if some node is not contained in the metrical tree, then the system as well as these nodes is not complete observability. DF only considers the "depth" through the process of expanding, which makes the 
observational topologies, lay over each other unavoidably and increases the unwanted redundancy.

\section{Proposed Approach for Voltage Stability Assessment}

The conventional method of computing loading margin $\lambda_{\max }$ is very time-consuming which limits its application for online. As an alternative, a fast and accurate real time voltage stability monitoring tool is proposed for estimation of loading margins. The voltage magnitudes and phase angles obtained from PMUs are given as the input of the ELM. Depth First (DF) method is used for placing the PMUs. To make the ELM approach applicable for a large scale power system problem, Mutual information (MI) based feature selection is proposed to achieve the input dimensionality reduction. MI-based feature selection reduces the number of network input features which reduces the network training time and improves the generalization capability. The various steps involved in the proposed approach for voltage stability assessment are given below:

\subsection{Selection of input and output variables}

The generalization capability of the ELM is highly dependent on the input variables that are selected for training the ELM model. Selection of appropriate input variables will help the network to improve its generalization performance. There are several power system state variables which can be fed as inputs to the ELM predictor network. In general, real and reactive power demand at load buses, line flows, bus voltage magnitudes and phase angles are chosen as the input variables. But for the real time applications, PMU data are used as input variables as they provide direct synchronized measurements of realtime phasors of voltages and currents. Therefore, load bus voltage magnitudes and phase angles obtained from PMUs are chosen as inputs attributes for the ELM model. Also, these selected variables can enhance the prediction accuracy of the network developed. Loading margins under contingency state are the outputs of the ELM network.

\subsection{Generation of input and output training data}

After identifying the network input and output variables, generation of sufficient input and output training data for the ELM are obtained using the following procedure:

- The input training data are the pre-contingency voltage magnitudes and phase angles at the load buses which are collected from PMUs.

- With the random variation in the real and reactive power at all the load buses from the base operating point and proportionate variation in the real power generation, the post - contingency loading margins are determined by carrying out off-line contingency analysis.

\subsection{Strategy for dimensionality reduction}

Dimensionality reduction technique is mandatory to reduce the input variables, thus reducing the measurement cost. If all the input variables are considered for training the developed network, then the system size grows bigger which results in large training time. To make the ELM approach applicable for a large scale power system problem, Mutual information (MI) -based feature selection is proposed to achieve the dimensionality reduction by selecting a subset of features from the initial set of available features thereby reducing the number of PMUs required. MI-based feature selection reduces the number of network input features which reduces the network training time and improves the generalization capability.

\subsection{Data normalization}

To avoid the dominance of higher valued input variables over the smaller ones and to prevent the saturation of simulation neuron, the input data are normalized by using the following expression:

$$
x_{n}=\frac{\left(x-x_{\min }\right) \times \text { range }}{\left(x_{\max }-x_{\min }\right)}+\text { starting value }
$$

where, $x_{n}$ is the normalized value and $x_{\min }$ and $x_{\max }$ are the minimum and maximum values of the data.

\subsection{Choice of network learning algorithm}

The proposed ELM learning algorithm is a new and promising algorithm with a lot of salient features which make it to be more superior to the conventional back propagation learning algorithm. Because of the slow gradient-based learning algorithms with all the parameters tuned iteratively, the training time of feed forward neural network is in general higher. With the ELM, single layer feed forward neural network can be simply considered as a linear system for mapping the input with the output that provides good generalization performance. Further, this kind of learning is faster which tends to reach small training error and weights.

\subsection{Network training and testing}

The normalized input features are presented to the ELM network for training and tested with a new input data, which is not previously used for training. The accuracy is evaluated by calculating the root mean square error (RMSE). Once the network is trained and tested, the developed network is ready for estimating the loading 
margin values at different operating conditions.

\section{MI-Based Feature Selection}

The mutual information between the input variables and the output provides the basis for feature selection. For feature selection, first the mutual information between each variable and the model output is calculated using Eq. (2)(5). If a variable has a high value of MI, then this variable provides a significant effect on the estimated output value. On the other hand, those variables with low values of MI will be regarded as minor effects on the output. Corresponding to each line outage the mutual information value of each input variable is calculated corresponding to the output and each variable is assigned a weightage based on the calculated value of mutual information. Then the first few variables with high overall weightage are selected to train this network.

\subsection{Algorithm of Mutual Information}

According to Shannon's information theorem, the random variable $Y$ uncertainty can be measured using entropy $H(Y)$. Thus, for these two variables $X$ and $Y$, conditional entropy $H(Y / X)$ measures the uncertainty of variable $Y$, when the variable $X$ is known. Thus, the mutual information $I(Y ; X)$ measures the certainty of the variable $Y$ by resolving the variable $X$. The relation between $H(Y), H(Y / X), I(Y ; X)$ is given by

$$
H(Y)=H(Y / X)+I(Y ; X)
$$

The main objective of proposing a training classification model is to reduce the uncertainty predictions on output variable $Y$, for the given input variable $X$. Thus, training a classifier is to improve the MI $I(Y ; X)$ as required. If $I(Y ; X)=0$ then the information contained in the observations may not be useful for determining the output Y. The goal is to achieve naturally the feature selection process for classification in order to obtain higher values with the smallest possible size of feature subsets. The prior entropy in the following section is defined based on Shannon capacity, which is defined as follows.

Consider a stochastic system with input variable $X$ and output variable $Y$. Let the discrete variable with variable $X$ has $N_{x}$ number of possible values and the variable $\mathrm{Y}$ has $N_{y}$ number of possible values. Now the initial uncertainty about the variable $\mathrm{Y}$ is defined by the entropy $H(Y)$,

$$
H(Y)=-\sum_{j=1}^{N_{y}} P\left(Y_{j}\right) \times \log \left(P\left(Y_{j}\right)\right.
$$

where $P\left(Y_{j}\right)$ is the probability of the different value of the variable $Y$. The remaining amount of uncertainty about the system output variable $Y$ after knowing the input variable $X$ is defined by its conditional entropy $H(Y / X)$,

$$
H(Y / X)=-\sum_{i=1}^{N_{x}} P\left(X_{i}\right) \times\left(\sum_{j=1}^{N_{y}} P\left(Y_{j} / X_{i}\right) \times \log \left(P\left(Y_{j} / X_{i}\right)\right)\right)
$$

where $P\left(Y_{j} / X_{i}\right)$ is the conditional probability for output variable $Y_{j}$ given the input variable $X_{i}$. Now the difference between $H(Y)-H(Y / X)$ represents the uncertainty of the system's output, which can be resolved by knowing the input. Thus from eqn. (2) we may thus write,

$$
I(Y ; X)=H(Y)-H(Y / X))
$$

Thus the mutual information is therefore the amount by which the input variables provided by $X$ reduces the number of uncertainties about the random variable represented by $Y$. Mutual information, also called as a symmetrical measure, which represents the information gained from the output variable $Y$ after observing the input variable $X$ is equal to the information gained about the variable $X$ after observing $Y$. For the contingency selection problem, variable $X$ at load buses refers to the real and reactive power loads and variable $Y$ represents the postcontingency loading margin.

\section{Review of Extreme Learning Machine}

The conventional Back Propagation (BP) learning algorithm suffers from slow convergence, local minima and over-fitting problems as it is a first order gradient method. It also involves too many parameters which are needed to be tuned randomly. Extreme learning machine (ELM) $[20,21]$ is a new and promising three-step tuning free learning algorithm used for training the single hidden layer feedforward neural networks (SLFNs). Empirical risk minimization theory is adopted in ELM. The whole learning process is done within a single iteration. The proposed algorithm is able to provide good generalization performance, robustness, controllability and fast learning rate. ELM is remarkably efficient and tends to reach a global optimum. The input weights and hidden layer biases of ELM can be assigned randomly. In ELM, the hidden nodes are randomly initiated and then fixed without iteratively tuning. The weights of the output layer are calculated using the Moore-Penrose (MP) generalized inverse. ELM uses non-differentiable or even discontinuous functions as an activation function. Different from traditional learning algorithms, the proposed learning algorithm not only tends to reach the smallest training error but also the smallest norm of weights. Therefore, the proposed learning algorithm tends to have good generalization performance for feedforward neural networks. 


\subsection{Implementation of ELM}

The output function of SLFNs with $\tilde{N}$ hidden neurons and activation function $g(x)$ for $N$ arbitrary distinct samples $\left(x_{j}, t_{j}\right)$ is given below:

$$
\sum_{i=1}^{\tilde{N}} \beta_{i} g\left(w_{i} \cdot x_{j}+b_{i}\right)=t_{j}, \quad j=1, \ldots, N
$$

where

$$
x_{j}=\left[\begin{array}{c}
x_{1}^{T} \\
\vdots \\
x_{N}^{T}
\end{array}\right] \in R^{n} \quad \text { and } t_{j}=\left[\begin{array}{c}
t_{1}^{T} \\
\vdots \\
t_{N}^{T}
\end{array}\right] \in R^{m}
$$

$x_{j}$ represent the $N \times n$ input vector which corresponds to the voltage magnitudes of the PMU located buses. $n$ is the number of input variables.

$t_{j}$ represent the $N \times m$ target vector which corresponds to the loading margins for the specified single line outages. $m$ is the number of output variables.

$w_{i}=\left[w_{i 1}, w_{i 2}, . ., w_{i n}\right]^{T}$ is the weight vector connecting the $\mathrm{i}^{\text {th }}$ hidden neuron and the input neurons, $\beta_{i}=$ $\left[\beta_{i 1}, \beta_{i 2}, \ldots, \beta_{i m}\right]^{T}$ is the weight vector connecting the $\mathrm{i}^{\text {th }}$ hidden neuron and the output neurons, $b_{i}$ is the threshold of the $\mathrm{i}^{\text {th }}$ hidden neuron and $t_{j}$ is the output value of $\mathrm{j}^{\text {th }}$ input sample. $w_{i} \cdot x_{j}$ denotes the inner product of $w_{i}$ and $x_{j}$.

Eq. (6) can be rewritten in compact form as below:

$$
H \beta=T
$$

$H$ is a non-square matrix and $w_{i}, b_{i}, \beta_{i}$ exist in case of single line feed network algorithm, such that

$$
H=\left[\begin{array}{cll}
g\left(w_{1} \cdot x_{1}+b_{1}\right) & \cdots & g\left(w_{\tilde{N}} \cdot x_{1}+b_{\tilde{N}}\right) \\
\vdots & \cdots & \\
g\left(w_{1} \cdot x N+b_{1}\right) & \cdots & g\left(w_{\tilde{N}} \cdot x_{N}+b_{\tilde{N}}\right)
\end{array}\right]_{N \times \tilde{N}}
$$

Unlike single line feed network mentioned in Eq. (8), where the input weights and the hidden layer biases need to be adjusted and to adjust it dynamically an activation function $g(x)$ is required and it has to be infinitely differentiable, here all the input variables need not to be adjusted and in fact that $\boldsymbol{w}_{\boldsymbol{i}}$ and $\boldsymbol{b}_{\boldsymbol{i}}$ are not necessarily need to be tuned and the output hidden layer matrix $\mathbf{H}$ can remain unchanged once if the variables are assigned randomly in the beginning of learning.

For hidden layer bias and fixed input weight $w_{i}$, seen from Eq. (8), to train a single line feed network is to find the least squares solution $\hat{\beta}$ of the linear system is

$$
\min _{\beta}\|H \beta-T\|
$$

If the number $N$ of distinct training samples is equal to the number of hidden nodes $\hat{N}$, such that $H$ becomes invertible and square matrix and when the hidden bias $\boldsymbol{b}_{\boldsymbol{i}}$ and the input weight vectors $w_{i}$ are chosen randomly, and this ELM training samples can approximated with zero error. However, if the number of hidden nodes is less than the training samples, then $H$ becomes a non-square matrix and now $w_{i}, b_{i}, \beta_{i}(i=1, \ldots, \hat{N})$ may not exist, such that $H \beta=T$. Finally, the above linear system is solved as smallest norm least squares which is the minimum norm least-squares solution which is shown as:

$$
\beta=H^{\dagger} T
$$

where $H^{\dagger}=\left(H^{T} H\right)^{-1} H^{T}$ is the Moore-Penrose generalized inverse of matrix $\mathrm{H}$

The steps of ELM algorithm are briefly given below:

Step 1: Random assignment of input weights and bias for the given activation function and hidden neurons.

Step 2: Calculation of the hidden layer output vector.

Step 3: Calculation of the output weight vector $\beta$ : $\beta=H^{\dagger} T$

\section{Simulation Results and Discussions}

IEEE 30-bus system is considered to demonstrate the effectiveness of the proposed methodology under various loading conditions considering and under single line contingency state. The test system considered has 6 generator buses, 24 load buses and 41 transmission lines. For this test system, based on the off-line contingency analysis, the severe contingencies are identified as 1-2, 2827, 27-30, 27-29, 2-5, 9-10 and 4-12. Depth First (DF) search algorithm is used for placing the PMUs. PMUs are located in such a way that they provide all the necessary measurements for the real time monitoring of entire power system voltage stability with the minimum number of measuring devices. The buses identified for placing the PMUs using the DF algorithm are 3, 5, 6, 11, 12, 17, 18, 20, 21, 24, 26 and 27 as shown in Fig. 2.

For real time voltage stability monitoring, the PMU data are used as input variables for the developed ELM model as they provide direct synchronized measurements of realtime phasors of voltages and currents. Therefore, load bus voltage magnitudes and phase angles obtained from PMUs by simulation results are chosen as inputs attributes for the ELM model. Also, these selected variables can enhance the prediction accuracy of the network developed. Loading margins under severe contingencies are chosen as the output variables.

After identifying the network input and output variables, generation of sufficient input and output training data for the ELM are obtained. The input training data are the precontingency bus voltage magnitudes and phase angles 


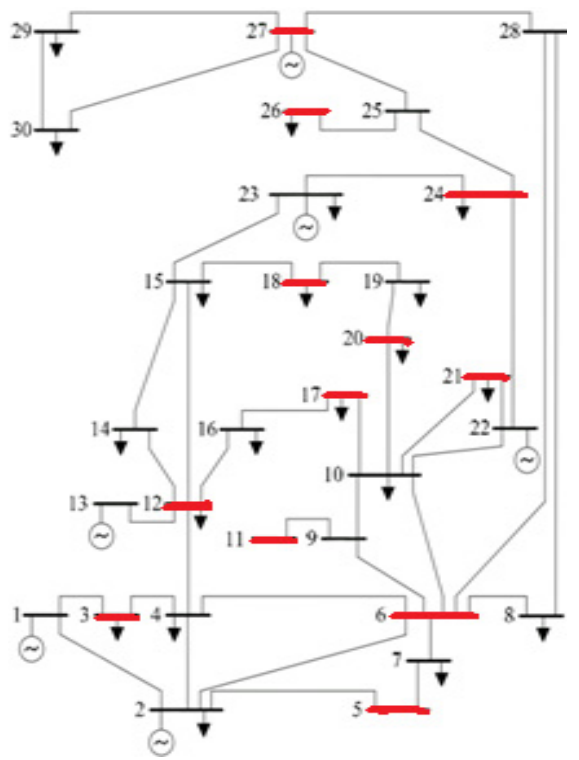

Fig. 2. IEEE 30 bus diagram for illustrating optimal PMU locations

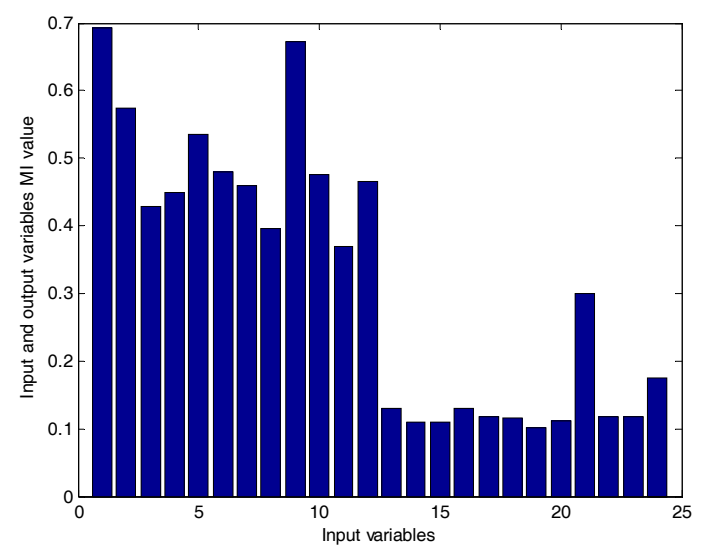

Fig. 3. Simulation result of MI-based feature selection

which are obtained as outputs from PMUs by simulation results. The loading margins for the identified severe contingencies are obtained for 1000 different loading conditions by randomly varying the real and reactive power at all the load buses from the base operating point with the proportionate variation in the real power generation by carrying out off-line contingency analysis at constant power factor. Out of 1000 input-output pairs, 750 data are taken for training and 250 for testing. The total number of input variables fed to the ELM network is 24 and the number of output variables is 7 which correspond to the loading margins of identified critical contingencies.

To make the ELM approach applicable for a large scale power system problem, Mutual information (MI) -based feature selection is proposed to achieve the dimensionality reduction thereby reducing the number of PMUs required. MI-based feature selection reduces the number of network input features which reduces the network training time
Table 1. Comparison of ELM performance with all features and with feature selection

\begin{tabular}{c|c|c}
\hline Parameters & $\begin{array}{c}\text { ELM with all } \\
\text { features }\end{array}$ & $\begin{array}{c}\text { ELM with feature } \\
\text { selection }\end{array}$ \\
\hline Number of input variables & 24 & 10 \\
\hline Number of output variables & 7 & 7 \\
\hline Training data & 750 & 750 \\
\hline Testing data & 250 & 250 \\
\hline Training accuracy (RMSE) & $8.9924 \mathrm{e}-006$ & $1.8727 \mathrm{e}-008$ \\
\hline Testing accuracy (RMSE) & $1.5351 \mathrm{e}-004$ & $9.3992 \mathrm{e}-005$ \\
\hline Training time (s) & 2.9063 & 2.8594 \\
\hline Testing time (s) & 0.0011 & 0.0011 \\
\hline
\end{tabular}

Table 2. Comparison of ELM output with CPF result

\begin{tabular}{c|c|c|c|c}
\hline \multirow{2}{*}{ Line outage } & \multicolumn{2}{|c|}{ ELM output } & \multicolumn{2}{c}{ CPF result } \\
\cline { 2 - 5 } & $\begin{array}{c}\text { Loading margin } \\
\text { in p.u }\end{array}$ & Rank & $\begin{array}{c}\text { Loading margin } \\
\text { in p.u }\end{array}$ & Rank \\
\hline $1-2$ & 0.2612 & I & 0.2612 & I \\
\hline $2-5$ & 2.0411 & V & 2.0410 & V \\
\hline $4-12$ & 2.7010 & VII & 2.7010 & VII \\
\hline $9-10$ & 2.4207 & VI & 2.4207 & VI \\
\hline $28-27$ & 1.4144 & II & 1.4144 & II \\
\hline $27-29$ & 2.0143 & IV & 2.0143 & IV \\
\hline $27-30$ & 1.8089 & III & 1.8089 & III \\
\hline
\end{tabular}

and improves the generalization capability. The simulation result of the proposed MI-based feature selection method is illustrated in Fig. 3, from which, it is evident that only a few variables are having high value of mutual information. Only, those input variables which have overall high mutual information values are selected to train the ELM. The selected features for the ELM model are only the voltage magnitudes at the buses $3,5,6,11,12,17,18$, 21,24 and 27.

To avoid the dominance of higher valued input variables over the smaller ones and to prevent the saturation of simulation neuron, the input data are normalized. The normalized input features are presented to the ELM network for training and tested with a new input data, which is not previously used for training. The accuracy is evaluated by calculating the root mean square error (RMSE). The performance comparison of ELM model without and with feature selection is given in Table 1.

Thus, it is very clear that the PMU data obtained by the simulation results are sufficient for the developed ELM model to estimate the post contingency loading margins accurately. Also, it is seen that the generalization performance of the ELM trained with the reduced input variables using MI-based feature selection method has been improved. Thus, only 10 input variables obtained from PMU are sufficient to train the ELM.

Table 2 shows the comparison of ELM output with CPF result for one particular loading condition along with ranking of contingencies.

The ELM results validate the proposed methodology for on line estimation of loading margin for large scale power system. The result shows that ELM developed can be 
Table 3. Performance comparison of ELM with BPNN

\begin{tabular}{c|c|c}
\hline Parameters & ELM & BPNN \\
\hline Number of input variables & 10 & 10 \\
\hline Number of output variables & 7 & 7 \\
\hline Training data & 750 & 750 \\
\hline Testing data & 250 & 250 \\
\hline Training accuracy (RMSE) & $1.8727 \mathrm{e}-008$ & 0.0090 \\
\hline Testing accuracy (RMSE) & $9.3992 \mathrm{e}-005$ & 0.0122 \\
\hline Training time (s) & 2.8594 & 48.25 \\
\hline Testing time (s) & 0.0011 & 0.1563 \\
\hline
\end{tabular}

used for fast and accurate contingency ranking. The performance comparison of proposed ELM with the conventional BPNN is shown in Table 3.

The results show that the training time of ELM is reduced compared to that of BP learning algorithm which makes the ELM an extremely fast learning network.

After training, the developed network is able to estimate the loading margin of severe contingencies within a short duration i.e., 0.0011 seconds when compared to time taken to compute loading margin of 7 line outages using conventional power flow method i.e., 12.575 seconds. This shows that the proposed ELM is computationally efficient and is suitable for on-line voltage stability assessment for multiple contingencies. Once the network is trained and tested, the developed network is ready for estimating the loading margin values at different operating conditions in real time using real time PMU measurements.

\section{Conclusion and Future Work}

In this paper, an ELM-based model is proposed for online voltage stability margin estimation. The proposed ELM-based online voltage stability monitoring tool has been proved to be an accurate and valid model for online estimation of power system loading margins for severe contingencies using the synchronized phasor measurements. Depth First (DF) method is used for placing the PMUs ensuring the complete observability of the power system with the minimum number of measuring devices connected to it. To make the ELM approach applicable for a large scale power system problem, Mutual information (MI) based feature selection is proposed to achieve the input dimensionality reduction. MI-based feature selection reduces the number of network input features which reduces the network time and improves the generalization capability. IEEE 30-bus test system is considered for a demonstration of effectiveness of the proposed methodology under various loading conditions considering single line contingencies. Simulation results validate the proposed ELM technique for fast and accurate online voltage stability assessment using PMU data. For future work, the voltage magnitude and phase angles of excluded buses where PMUs have not been installed can be reconsidered using Mutual Information (MI)- based feature selection method inorder to identify those input features which are having high value of mutual information so that network accuracy can be further enhanced. Also, it is planned to implement Genetic Algorithm (GA) and other optimization algorithms for optimal placement of PMU in future.

\section{References}

[1] IEEE Special Publication, 90TH0358-2-PWR, "Voltage Stability of Power Systems: Concepts, Analytical Tools and Industry Experience," 1990.

[2] Tiranuchit and R. J. Thomas, "A Posturing Strategy Against Voltage Instabilities in Electric Power Systems," IEEE Transactions on Power Systems, Vol. 3, No. 1, pp. 87-93, Feb. 1998.

[3] P.A. Löf, T Smed, G. Anderson and D. J. Hill, "Fast Calculation of a Voltage Stability Index," IEEE Transactions on Power Systems, Vol. 7, No. 1, pp. 54-64, Feb. 1992.

[4] P. Kessel and H. Glavitsch, "Estimating the Voltage Stability of Power Systems," IEEE Transactions on Power Systems, Vol.1, No.3, pp. 346-354, July 1986.

[5] Gao. G.K. Morison and P. Kundur, "Voltage Stability Evaluation using Modal Analysis," IEEE Transactions on Power Systems, Vol. 7, No. 4, pp. 15291542, Nov. 1992.

[6] P.A. Lof, G. Anderson and D.J. Jill, "Voltage Stability Indices for Stressed Power System," IEEE Transactions on Power Systems, Vol. 8, No. 1, pp. 326-335, Feb. 1993.

[7] C.A. Canizares, A.Z. de Souza and V.H. Quintana, "Comparison of Performance Indices for Detection of Proximity to Voltage Collapse," IEEE Transactions on Power Systems, Vol. 11, No. 3, pp. 1441-1450, August 1996.

[8] C.A. Canizares, F.L. Alvarado, C.L. DeMarco, I. Dobson and W.F. Long, "Point Of Collapse Methods Applied to Ac/Dc Power Systems," IEEE Transactions on Power Systems, Vol. 7, No. 2, pp. 673-683, May 1992.

[9] V. Ajjarapu and C. Christy, "The Continuation Power Flow: A Tool for Steady State Voltage Stability Analysis," IEEE Transactions on Power Systems, Vol. 7, No.1, pp. 416-423, Feb. 1992.

[10] G. K. Morison, B. Gao, and P. Kundur, "Voltage Stability Analysis using Static and Dynamic Approaches," IEEE Transactions on Power Systems, Vol. 8, No. 3, pp. 1159-1165, August 1993.

[11] M.K.Pal, "Voltage Stability Conditions Considering Load Characteristics," IEEE Transactions on Power Systems, Vol. 7, No. 1, pp. 243-249, Feb. 1992.

[12] Karlsson and D. J. Hill, "Modeling and Identification of Nonlinear Dynamic Loads in Power Systems," IEEE Transactions on Power Systems, Vol. 9, No. 1, pp. 157-163, Feb. 1994. 
[13] D. Devaraj, J. Preetha Roselyn and R. Uma Rani, "Artificial Neural Network Model for Voltage Security Based Contingency Ranking," Applied Soft Computing, Vol. 7, No. 3, pp. 722-727, June 2007.

[14] S. Chakrabarti, "Voltage Stability Monitoring by Artificial Neural Network using a Regression-Based Feature Selection Method," Expert Systems with Applications, Vol. 35, No. 4, pp. 1802-1808, Nov. 2008.

[15] D. Devaraj, B. Yegnanarayana and K. Ramar, "Radial Basis Function Networks for Fast Contingency Ranking," Electric Power and Energy Systems Journal, Vol. 24, No. 5, pp. 387-395, June 2002.

[16] Saikat Chakrabarthi, and Benjamin Jeyasurya, "MultiContingency Voltage Stability Monitoring of a Power System using an Adaptive Radial Basis Function Network," Electric Power and Energy Systems Journal, Vol. 30, No.1, pp. 1-7, Jan. 2008.

[17] Jayashankar V, Kamaraj N, and Vanaja N, "Estimation of Voltage Stability Index for Power System Employing Artificial Neural Network Technique and TCSC Placement," Neurocomputing, Vol. 73, No. 1683, pp. 3005-3011, Oct. 2010.

[18] D. Devaraj and J. Preetha Roselyn, "On-Line Voltage Stability Assessment using Radial Basis Function Network Model with Reduced Input Features," Electrical Power and Energy Systems Journal, Vol. 33, No. 9, pp: 1550-1555, Nov. 2011.

[19] P. Aravindhababu, G. Balamurugan, "ANN based Online Voltage Estimation," Applied Soft Computing, Vol. 12, No.1, pp. 313-319, Jan. 2012.

[20] T. L. Baldwin, L. Mili, M. B. Boisen, Jr, and R. Adapa, "Power System Observability with Minimal Phasor Measurement Placement," IEEE Trans. Power Syst., Vol. 8, No. 2, pp. 707-715, May 1993.

[21] Guang-Bin Huang, Qin-Yu Zhu and Chee-Kheong Siew, "Extreme Learning Machine: Theory and Applications," Neurocomputing, Vol. 70, No. 1-3, pp. 489-501, Dec. 2006.

[22] Guang-Bin Huang, Hongming Zhou, Xiaojian Ding, and Rui Zhang, "Extreme Learning Machine for Regression and Multiclass Classification," IEEE Transactions on Systems, Man, and Cybernetics, Vol. 42, No.2, pp. 513-529, April 2012.

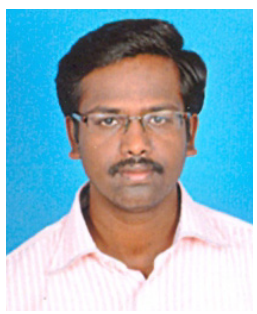

P. Duraipandy He completed his B.E and M.E in Electrical \& Electronics Engineering and Power System Engineering in the year 2004 and 2006, respectively, from K.L.N College of Engineering, Madurai and Arulmigu Kalasalingam College of Engineering, Krishnankoil. He is pursuing Ph.D in Kalasalingam University. He worked as Assistant Professor in the Electrical \& Electronics Engineering department of Kalasalingam University, Krishnankoil from May 2007 to June 2011. Since June 2011, he is working as a faculty in the Electrical \& Electronics Engineering department of Velammal College of Engineering and Technology, Madurai. He has published 6 papers in Journals and presented 25 papers in conferences. His research interest includes Power system voltage stability analysis, Smart grid, Evolutionary algorithms and Neural networks.

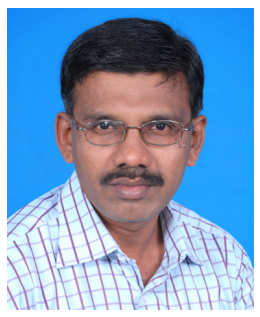

D. Devaraj D. Devaraj completed his B.E and M.E in Electrical \& Electronics Engineering and Power System Engineering in the year 1992 and 1994, respectively, from Thiagarajar College of Engineering, Madurai. From 1994 to 1997, he worked as a Lecturer in Arulmigu Kalasalingam College of Engineering, Krishnankoil. He obtained his Ph.D degree from IIT Madras, Chennai in the year 2001. Since 2001, he is working as a faculty in the Electrical \& Electronics Engineering department of Kalasalingam University. $\mathrm{He}$ has organized 6 Conferences, 8 Seminars and conducted 10 workshops. He has authored 2 text books, Power system analysis and Power system control. He has published 105 papers in Journals and presented 250 papers in conferences. He has chaired 15 technical sessions in various National and International Conferences. He is the Editorial board member of the International Journal of Adaptive and Innovative Systems and reviewer of IEEE Transaction on Fuzzy System, IEEE Transaction on System, Man, Cybernatics, IET Proceedings on Generation, Transmission \& Distribution, International Journal on Electric Power \& Energy Systems, Electric Power Components and Systems, Neuro computing and Applied Soft computing Journal. He has Supervised 15 Ph.D, 2 M.S and 25 M.E thesis. Presently, he is guiding 8 Ph.D scholars. He is the Project Director of Power System Automation Group attached to TIFAC-CORE in Network Engineering which is sponsored by DST, Government of India and Principal Investigator of the DST funded research project on "Development of Maximum power point tracking algorithm for Solar PV system". His research interest includes Power system security, Voltage stability, Smart grid, Evolutionary algorithms, Neural network and Data Mining. He is a senior member IEEE. Presently, he is the Professor \& $\mathrm{HoD} / \mathrm{EEE}$ at Kalasalingam University, Krishnankoil. 